\title{
Characterizing the Reserve Provision Capability Area of Active Distribution Networks: A Linear Robust Optimization Method
}

\author{
Mohsen Kalantar-Neyestanaki ${ }^{(}$, Student Member, IEEE, Fabrizio Sossan $\left.{ }^{(}\right)$, Member, IEEE, \\ Mokhtar Bozorg ${ }^{(}$, Member, IEEE, and Rachid Cherkaoui, Senior Member, IEEE
}

\begin{abstract}
Distributed energy resources (DERs) installed in active distribution networks (ADNs) can be exploited to provide both active and reactive power reserves to the upper-layer grid (i.e., sub-transmission and transmission systems) at their connection point. This paper introduces a method to determine the capability area of an ADN for the provision of both active and reactive power reserves while considering the forecast errors of loads and stochastic generation, as well as the operational constraints of the grid and DERs. The method leverages a linearized load flow model and introduces a set of linear scenario-based robust optimization problems to estimate the reserve provision capability (RPC) area of the ADN. It is proved that, under certain assumptions, the RPC area is convex. The performance of the proposed method is tested on a modified version of the IEEE 33-bus distribution test system.
\end{abstract}

Index Terms-Active distribution network (ADN), distributed energy resources (DERs), flexibility, reserve provision capability (RPC) curve, reserve provision capability (RPC) area, scenariobased robust optimization, TSO/DSO interface.

\section{NOMENCLATURE}

\section{Indices and Sets}

$0^{\prime}$

$f$

$h$

$i, j$
Index for the common coupling node between the ADN and the upper-layer grid.

Index for the linear boundaries modeling the maximum current flow limit of a branch. Index for dispatchable distributed generators (DDGs).

Manuscript received November 18, 2018; revised April 5, 2019 and September 6, 2019; accepted November 3, 2019. Date of publication November 28, 2019; date of current version April 21, 2020. This work was supported by the Swiss Centre for Competence in Energy Research on the Future Swiss Electrical Infrastructure through the Swissgrid and the Swiss Innovation Agency (Innosuisse-SCCER Program). Paper no. TSG-01773-2018. (Corresponding author: Mohsen Kalantar-Neyestanaki.)

M. Kalantar-Neyestanaki and R. Cherkaoui are with the Power Systems Group, École Polytecnique Fédérale de Lausanne, 1015 Lausanne, Switzerland (e-mail: mohsen.kalantar@epfl.ch; rachid.cherkaoui@epfl.ch).

F. Sossan is with the Center for Processes, Renewable Energies and Energy Systems, Mines ParisTech, Paris, France (e-mail: fabrizio.sossan@mines-paristech.fr).

M. Bozorg is with the School of Management and Engineering Vaud (HEIG-VD), University of Applied Sciences Western Switzerland, 1401 Yverdon-les-Bains, Switzerland (e-mail: mokhtar.bozorg@heig-vd.ch).

Color versions of one or more of the figures in this article are available online at http://ieeexplore.ieee.org.

Digital Object Identifier 10.1109/TSG.2019.2956152 $k$

$l$

$n$

$s$

$t$

$\mathbb{B}$

$\mathbb{D G}$

$\mathbb{D G}_{i}$

$\mathbb{F} l$

$\mathbb{L}$

$\mathrm{N}_{\mathbb{B}}$

$\mathrm{N}^{\mathrm{DG}}, \mathrm{N}^{\mathrm{SG}}$

$\mathrm{N}_{S}$

$\mathbb{C}_{h}, \mathbb{C}_{k}$

$\mathbb{S G}_{i}$

$\mathbb{S}$

$\psi$

$\Omega(\psi), \Gamma(\psi)$ Set of inequality/equality constraints modeling the operational constraints of ADN and DERs.

Index for stochastic distributed generators (SDGs).

Index for branches.

Index for the linear boundaries modeling the capability curve of a DDG/SDG.

Index for scenarios.

Index for time intervals.

Set of nodes of ADN excluding $0^{\prime}$.

Set of DDGs of ADN.

Set of DDGs connected to node $i$.

Set of the linear boundaries modeling the maximum current flow limit of branch $l$.

Set of branches of ADN.

Number of nodes of ADN excluding $0^{\prime}$. Number of DDGs/SDGs installed in ADN. Number of scenarios belonging to set $\mathbb{S}$. Set of linear boundaries modeling the capability curve of DDG $h /$ SDG $k$.

Set of SDGs connected to node $i$.

Set of selected credible scenarios.

Set of optimization variables.
Variables

$I_{l t s}$

$I_{l t s}^{\mathrm{real}}, I_{l t s}^{\mathrm{imag}}$

$\left|I_{l t s}\right|$

$r_{0^{\prime} t s}^{\mathrm{P}}, r_{0^{\prime} t s}^{\mathrm{Q}}$

$r_{h t s}^{\mathrm{DG}, \mathrm{P}}, r_{h t s}^{\mathrm{DG}, \mathrm{Q}}$

$r_{k t s}^{\mathrm{SG}, \mathrm{Q}}$

$R_{0^{\prime} t}^{\mathrm{P}}, R_{0^{\prime} t}^{\mathrm{Q}}$

\footnotetext{
Current phasor of branch $l$ during time slot $t$ and scenario $s$ [p.u.].

Real/imaginary part of $I_{l t s}[p . u$.$] .$

Magnitude of $I_{l t s}[p . u$.].

Active/reactive power reserve absorbed by ADN from the upper-layer grid during time slot $t$ and scenario $s$ [p.u.].

Active/reactive power reserve injected by DDG $h$ to the grid during time slot $t$ and scenario $s[p . u$.$] .$

Reactive power reserve injected by SDG $k$ to the grid during time slot $t$ and scenario $s[p . u$.$] .$

Active/reactive power reserve capacity absorbed by ADN from the upper-layer grid during time slot $t$ [p.u.].
} 
$P_{0^{\prime} t s}^{\mathrm{Net}}, Q_{0^{\prime} t s}^{\mathrm{Net}}$

Total active/reactive power absorbed by $\mathrm{ADN}$ at its connection point during time slot $t$ and scenario $s$ [p.u.].

$\Delta P_{i t s}^{\mathrm{Net}}, \Delta Q_{i t s}^{\mathrm{Net}} \quad$ Net deviation of the active/reactive power injection at node $i$ from its base case operating point (i.e., scheduled operating point) during time slot $t$ and scenario $s$ [p.u.].

$V_{j t s}$ Voltage magnitude of node $j$ during time slot $t$ and scenario $s$ [p.u.].

\section{Parameters}

$\mathrm{a}^{\mathrm{P}}, \mathrm{a}^{\mathrm{Q}}$

$\mathbf{E}_{j t}^{0}, \mathbf{E}_{j i t}^{\mathrm{P}}$,

$\mathbf{E}_{j i t}^{\mathrm{Q}}$

$\mathbf{F}_{f l}^{0}, \mathbf{F}_{f l}^{\mathrm{real}}$,

$\mathbf{F}_{f l}^{\mathrm{imag}}$

$\mathbf{G}_{t}^{\mathrm{P} 0}, \mathbf{G}_{i t}^{\mathrm{PP}}$,

$\mathbf{G}_{i t}^{\mathrm{PQ}}$

$\mathbf{G}_{t}^{\mathrm{Q} 0}, \mathbf{G}_{i t}^{\mathrm{QP}}$,

$\mathbf{G}_{i t}^{\mathrm{QQ}}$

$\mathbf{H}_{l t}^{0, \text { real }}, \mathbf{H}_{l i t}^{\mathrm{P}, \text { real }}$,

$\mathbf{H}_{\text {lit }}^{\mathrm{Q} \text {,rea }}$

$\mathbf{H}_{l t}^{0, \text { imag }}, \mathbf{H}_{l i t}^{\mathrm{P}, \mathrm{imag}}$

$\mathbf{H}_{\text {lit }}^{\mathrm{Q} \text {,imag }}$

$\mathrm{I}_{l}^{\mathrm{Max}}$

$\mathbf{K}_{n h t}^{0}$,

$\mathbf{K}_{n h}^{\mathrm{P}}, \mathbf{K}_{n h}^{\mathrm{Q}}$

$\mathbf{M}_{n k t s}^{0}, \mathbf{M}_{n k t s}^{\mathrm{P}}$,

$\mathbf{M}_{n k t s}^{\mathrm{Q}}$

$\mathrm{P}_{0^{\prime} t}^{\text {Base }}, \mathrm{Q}_{0^{\prime} t}^{\text {Base }}$

$\mathrm{P}_{i t}^{\text {Base }}, \mathrm{Q}_{i t}^{\text {Base }}$

$\Delta \mathrm{P}_{i t s}^{\mathrm{D}}, \Delta \mathrm{Q}_{i t s}^{\mathrm{D}}$

$\Delta \mathrm{P}_{k t s}^{\mathrm{SG}}$
Constant terms defining the search direction in $R_{0^{\prime} t}^{\mathrm{P}}-R_{0^{\prime} t}^{\mathrm{Q}}$ coordinate plane.

Constant terms of the linear function expressing $V_{j t s}$ based on $\Delta P_{i t s}^{\mathrm{Net}}$ and $\Delta Q_{i t s}^{\mathrm{Net}}$.

Constant terms of the linear functions $\left(f \in \mathbb{F}_{l}\right)$ modeling the maximum current flow limit of branch $l$ based on $I_{l t s}^{\text {real }}$ and $I_{l t s}^{\text {imag }}$. expressing $r_{0^{\prime} t s}^{\mathrm{P}}$ based on $\Delta P_{i t s}^{\mathrm{Net}}$ and $\Delta Q_{i t s}^{\mathrm{Net}}$.

Constant terms of the linear function expressing $r_{0^{\prime} t s}^{\mathrm{Q}}$ based on $\Delta P_{i t s}^{\mathrm{Net}}$ and $\Delta Q_{i t s}^{\mathrm{Net}}$

Constant terms of the linear function expressing $I_{l t s}^{\mathrm{real}}$ based on $\Delta P_{i t s}^{\mathrm{Net}}$ and $\Delta Q_{i t s}^{\mathrm{Net}}$.

, Constant terms of the linear function expressing $I_{l t s}^{\mathrm{imag}}$ based on $\Delta P_{i t s}^{\mathrm{Net}}$ and $\Delta Q_{i t s}^{\mathrm{Net}}$

Maximum current limit of the branch l[p.u.].

Constant terms of the linear functions $\left(n \in \mathbb{C}_{h}\right.$ ) modeling the capability curve of DDG $h$ based on $r_{h t s}^{\mathrm{P}, \mathrm{DG}}$ and $r_{h t s}^{\mathrm{Q}, \mathrm{DG}}$.

Constant terms of the linear functions $\left(n \in \mathbb{C}_{k}\right.$ ) modeling the capability curve of SDG $k$ based on $\Delta \mathrm{P}_{k t s}^{\mathrm{SG}}$ and $r_{k t s}^{\mathrm{Q}, \mathrm{SG}}$.

Active/reactive power absorbed by ADN at its connection point during time slot $t$ for the base case operating point (i.e., scheduled operating point) [p.u.].

Total active/reactive power injected at node $i$ during time slot $t$ for the base case operating point (i.e., scheduled operating point) $[p . u$.$] .$

Deviation of the active/reactive power consumption of the load connected to node $i$ from its base case operating point (i.e., scheduled operating point) during time slot $t$ and scenario $s$ [p.u.].

Deviation of the active power generation of SDG $k$ from its base case operating point (i.e., scheduled operating point) during time slot $t$ and scenario $s$ [p.u.].
Constant terms of the linear function

$\mathrm{V}_{j}^{\mathrm{Max}}$
$\mathrm{V}_{j}^{\mathrm{Min}}$
$\mathrm{t}_{0}$

$\mathrm{~T}$
$\tau$
$\theta$

$\tau$

\section{A.}

$\mathbf{E}$ Elvitions renewable energy technologies are leading towards replacing centralized conventional generation in favor of decentralized renewable generation [1]. The main challenge in the way of this transition is the increasing demand for controllable resources to be deployed to guarantee the frequency stability, voltage regulation, power quality, and congestion management, while the magnitudes of conventional ancillary services providers are decreasing [2]-[4].

On the other hand, the number of distributed energy resources (DERs) is progressively increasing in active distribution networks (ADNs). A promising solution to preserve the quality/security of supply is aggregating the flexibility of DERs located in ADNs to provide active/reactive power reserves to the upper-layer grid [5]-[8]. In this emerging architecture, further cooperation between operators of different levels of electric power systems, e.g., transmission system operators (TSOs) and distribution system operators (DSOs), is required to exchange flexibility [9]. Flexibility can be perceived as a service, like active/reactive power reserves, that a resource provides to the grid by adjusting its operating point [7], [10]. The question that arises is how much are the maximum active/reactive power reserves an $\mathrm{ADN}$ can provide upon request at its connecting point to the upper-layer grid? In this context, the reserve provision capability (RPC) curve of an ADN is defined as a curve characterizing the extreme amount of active and reactive power reserves that ADN can provide to the upper-layer grid. The area surrounded by the RPC curve is called RPC area. This area is a function of the ADN's operating point, ADN's grid constraints (i.e., voltage and current constraints) and the capability limits of DERs located in the ADN.

\section{B. Literature Review}

In [5], a random sampling approach is proposed to determine the RPC area of an ADN. It first generates random points inside the capability curve of each DERs and then, for each combination, applies a nonlinear load flow to determine the nodal voltages and lines currents. If they respect all grid constraints, the resulting power flow at the grid connection point is retained and used to compose the RPC area, otherwise it is discarded. The final output of this method is a set of feasible points instead of the perimeter of the ADN's RPC 


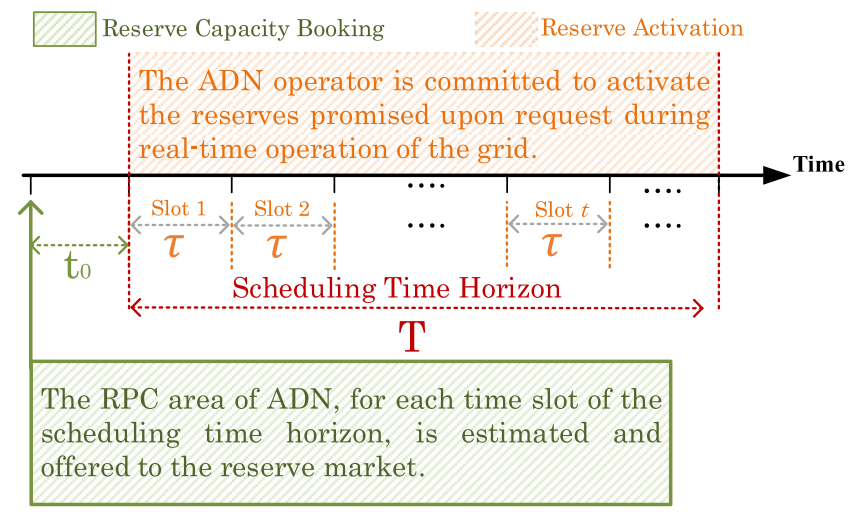

Fig. 1. The general procedure for estimating the ADN's RPC area.

area (RPC curve). This approach is essentially an exhaustive search, therefore, its computational complexity scales poorly with the number of DERs in the ADN.

Works in [6]-[8] propose to estimate the RPC curve of an ADN by solving a non-convex non-linear optimal power flow (OPF) problem. In comparison with the random sampling approach, OPF-based methods are better suited to identify accurately the full RPC area while might require less computational effort. However, since OPFs are notoriously non-convex, also this approach lacks guaranteed tractability properties.

In general, all the above-mentioned approaches suffer from the following limitations:

- they do not model uncertainties. As known, renewable generation and demand at high level of disaggregation is highly volatile. Consequently, these methods can only forecast the RPC area of ADN for short-term horizons (i.e., few minutes ahead), where uncertainties can be neglected. As typical power system operations entail scheduling operation on longer time horizons (e.g., dayahead and hours-ahead), accounting for uncertainties is a key to achieve a reliable estimation of the RPC;

- they model the RPC area of DERs as a rectangular area, which might be unrealistic;

- they estimate the RPC area by solving a non-convex optimization problem. Thus, they cannot mathematically ensure the global optimality;

- they entail a high computational effort when the number of DERs is large.

\section{Contributions and Organization of the Paper}

The limitations found in [5]-[8] inspired the contribution of this paper. It consists in a tractable algorithm based on a linear scenario-based robust optimization problem to estimate the curve and the area of the RPC of an ADN while considering grid constraints and uncertainties of loads and stochastic generation. Grid constraints are modelled by leveraging recent advancements in linearized load flow models [11], whereas uncertainty of loads and stochastic generation are explicitly modelled with scenarios, which are used to enforce robust grid constraints and deliver realistic estimates of the RPC area. To comply with the current scheduling/operation paradigm of current power systems, the proposed method is applied to a long-term scheduling horizon $\mathrm{T}$ (e.g., day-ahead), where uncertainties play a salient role. This longer-term horizon method, compared to existing ones, entails considering a large number of scenarios to model the forecasting errors of demand and stochastic generation. It is shown that the proposed method can compute robust estimates of the RPC of ADNs in a reliable and efficient way.

The rest of this paper is organized as follows. Section II states the problem. Section III describes the procedure to estimate the RPC curve of the ADN. Section IV develops the formulation of the estimation process based on a scenariobased robust linear optimization problem. Section $V$ introduces three theorems used to derive properties of the proposed method. Section VI introduces a straight-forward approach for determining the number of scenarios that should be considered in the problem introduced in Section IV. Section VII proposes a proof-of-concept by simulations on the IEEE 33-bus distribution test system. Finally, Section VIII summarizes the main conclusions.

\section{Problem Statement}

Although specifications might vary from system to system, the procedure to manage (book/activate) power reserve in electrical grids generally consists in two steps:

1- Reserve capacity booking (planning): This step is usually carried out ( $t_{0}$ hours) prior to the real-time grid operations. For each time slot of the scheduling time horizon T, the TSO estimates its needs for reserves, and reserve providers estimate their capacity for providing reserve. Then, demand and offers are collected in the reserve capacity market and the TSO books its required reserves by clearing the market. In this stage, no real product is exchanged between the TSO and reserve providers.

2- Reserve activation (real-time operation): This step is carried out during the real-time operation. In case of need, the TSO sends its requests to the reserve providers who already succeeded to sell their reserve capacities in the reserve capacity market. Then, those reserve providers activate all or a portion of the reserve capacities they have sold.

This paper focuses on the first step, namely reserve capacity booking. In line with the above-mentioned procedure, the method of this paper estimates the RPC area of an ADN for each individual time slot of the scheduling time horizon so that it can be offered to the reserve capacity market, according to the sequence of operation shown in the timeline of Fig. 1. It is considered that:

- The RPC area of an ADN for each time slot of the scheduling time horizon $\mathrm{T}$ is estimated $t_{0}$ hours before the beginning of the scheduling time horizon;

- The scheduling time horizon $\mathrm{T}$ consists of a number of time slots, each with duration of $\tau$;

- The ADN's forecasted operating point, i.e., $\mathrm{P}_{0^{\prime} t}^{\mathrm{Base}}$, $\mathrm{Q}_{0^{\prime} t}^{\text {Base }}, \mathrm{P}_{i t}^{\text {Base }}$ and $\mathrm{Q}_{i t}^{\text {Base }}$ for each time slot is assumed to be known and it is called ADN's base case operating point.

This method is general and can accommodate any desired scheduling horizon with any duration of time slots by 


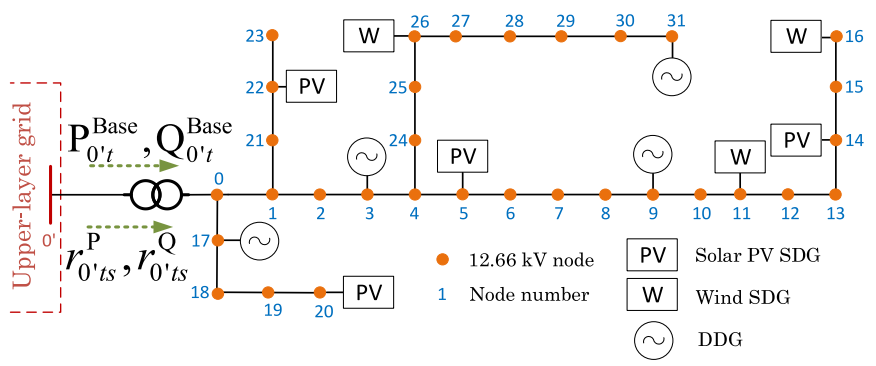

Fig. 2. The modified IEEE 33-bus distribution test system.

plugging-in suitable forecasts. Finally, it is worth noting that the RPC areas at various time slots are determined separately and in the same manner. Since there is no time-coupling constraint in the problem, the RPC areas are independent from each other. For this reason, the formulation in the next section refers to one time slot only, and it is applied identically to each time slot of the time horizon.

Although references to the distribution grid of Fig. 2 are made throughout the paper, the proposed formulation can accommodate any kind of grid (i.e., meshed or radial) with a single connection point to the upper-layer grid.

\section{MethoD}

The power flow at the connection point of the ADN in Fig. 2 is:

$$
\begin{aligned}
& P_{0^{\prime} t s}^{\mathrm{Net}}=\mathrm{P}_{0^{\prime} t}^{\mathrm{Base}}+r_{0^{\prime} t s}^{\mathrm{P}}, \\
& Q_{0^{\prime} t s}^{\mathrm{Net}}=\mathrm{Q}_{0^{\prime} t}^{\text {Base }}+r_{0^{\prime} t s}^{\mathrm{Q}} .
\end{aligned}
$$

It is assumed that $\mathrm{P}_{0^{\prime} t}^{\mathrm{Base}}$ and $\mathrm{Q}_{0^{\prime} t}^{\mathrm{Base}}$ are known, then, this method estimates the RPC curve, i.e., the boundary of $r_{0^{\prime} t s}^{\mathrm{P}}$ and $r_{0^{\prime} t s}^{\mathrm{Q}}$, while considering grid constraints and uncertainties of loads and stochastic generation.

The proposed method consists of three main parts: definition of the search directions, computation of the points of the RPC curve along the defined search directions, and estimation of the whole RPC curve. The three steps are explained in the following by referring to Fig. 3, which exemplifies the RPC on the $R_{0^{\prime} t}^{\mathrm{P}}-R_{0^{\prime} t}^{\mathrm{Q}}$ plane.

1- Definition of the search directions: As shown in Fig. 3, the angle $\theta$ defines a search direction in the $R_{0^{\prime} t}^{\mathrm{P}}-R_{0^{\prime} t}^{\mathrm{Q}}$ plane and can take a value between $0^{\circ}$ and $360^{\circ}$. It determines ap and $\mathrm{a}_{\mathrm{Q}}$ as follows:

$$
\begin{aligned}
& a_{p}= \begin{cases}-1 & 0^{\circ} \leq \theta<90^{\circ} \\
+1 & 90^{\circ}<\theta<270^{\circ} \\
-1 & 270^{\circ}<\theta<360^{\circ},\end{cases} \\
& \mathrm{a}_{\mathrm{Q}}=\tan (\theta) \quad 0^{\circ} \leq \theta<360^{\circ} .
\end{aligned}
$$

aP and aQ are used in the second step, when solving the linear robust optimization problem. A set of search directions is defined with the following procedure:

A. The minimum acceptable granularity for the final estimated RPC curve is defined. It determines the procedure termination criterion. The procedure is terminated when the Euclidean distances between two consecutive estimated points on the RPC curve

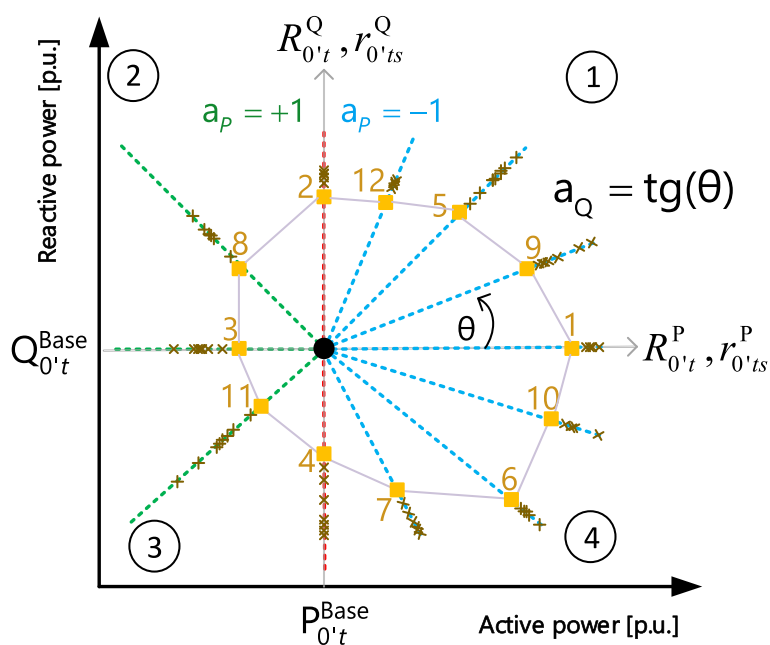

- Base case operating point.

- Maximum capability of $\mathrm{ADN}$ for provision of active/reactive power reserves in the selected direction over all scenarios $\left(R_{0, t}^{\mathrm{P}} / R_{0^{\prime} t}^{\mathrm{Q}}\right)$

$\times \quad$ Maximum capability of ADN for provision of active/reactive power reserves in the selected direction for an individual scenario $\left(r_{0, t s}^{\mathrm{P}} / r_{0^{\prime} t s}^{\mathrm{Q}}\right)$.

Selected search direction in quadrant 1 and 4.

Selected search direction in quadrant 2 and 3.

....... Selected search direction corresponding to $\theta= \pm 90^{\circ}$.

(1) Number of quadrant

Estimated RPC curve of ADN.

Fig. 3. Typical estimated RPC area of an ADN.

(yellow squares in Fig. 3) are smaller than the predefined granularity.

B. Four search directions, corresponding to $\theta=$ $0^{\circ}, 90^{\circ}, 180^{\circ}, 270^{\circ}$, are defined. For each search direction, the linear robust optimization problem introduced in step 2 is solved, determining 4 points on the RPC curve.

C. The search directions are ordered based on their increasing $\theta$.

D. The Euclidean distance between each couple of consecutive points for increasing $\theta$ on the RPC curve is calculated. If all the distances are smaller than the defined granularity in A, the procedure ends and goes to final step 3; otherwise, the couple of consecutive points with the largest distance is selected, and the arithmetic mean of their search direction $\theta$ is used as the new search direction.

E. For the new search direction in $\mathrm{D}$, the linear robust optimization problem of step 2, is solved.

F. Jump to $\mathrm{C}$.

The abovementioned procedure is illustrated in Fig. 3, where the numbers on the RPC curve denote the sequence of the defined directions.

2- Computation of the points of the RPC curve along the defined search directions: For a defined $\theta$, this step entails solving the optimization problem detailed in Section IV to find the point on the RPC curve associated to that search direction.

3- Estimation of the whole RPC curve: Once the points of the RPC curve are defined, they are linearly interpolated to approximate the whole RPC curve, as shown in 


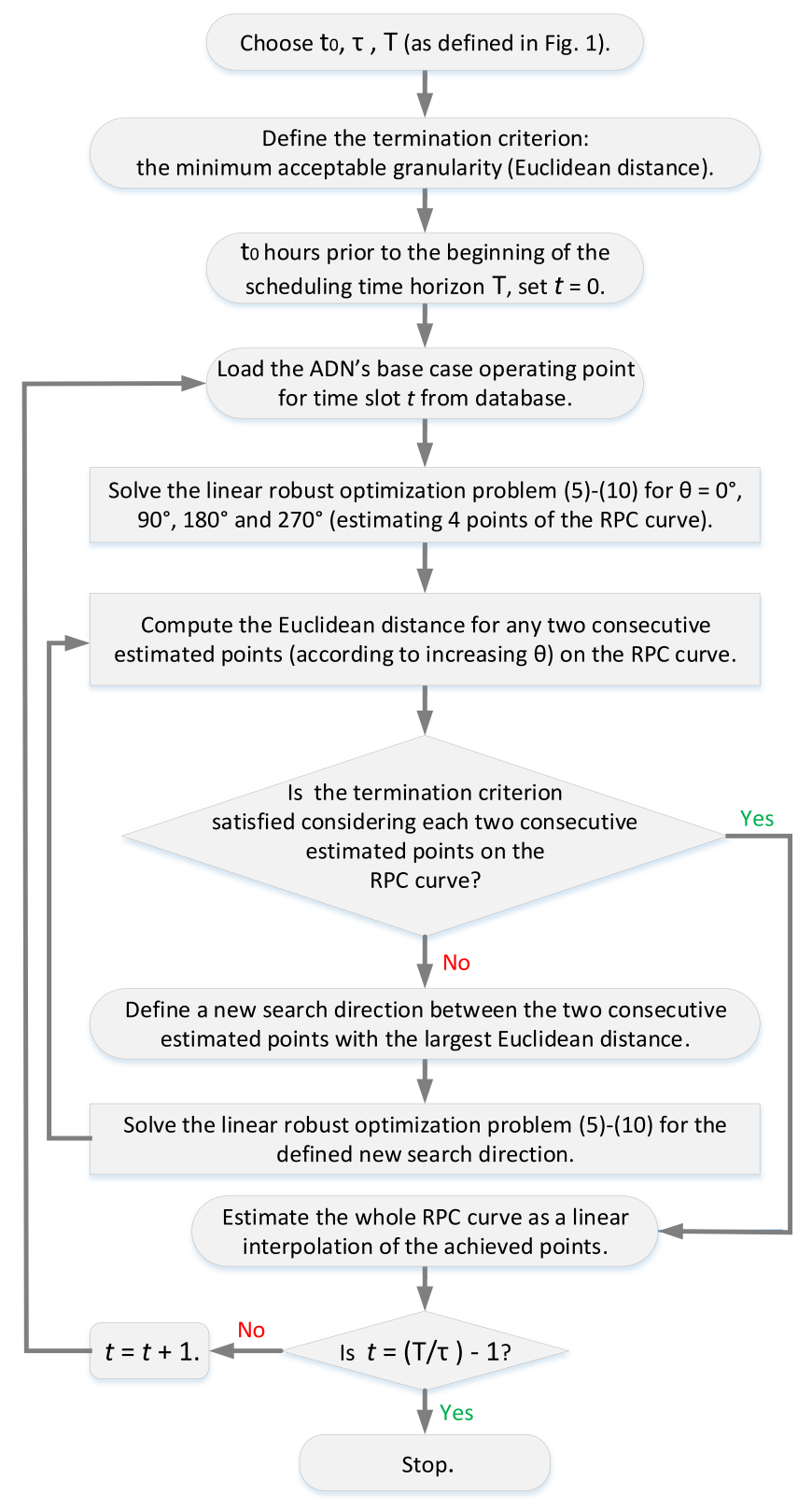

Fig. 4. Flowchart showing the procedure for estimating the RPC area of ADN for each time slot of the scheduling time horizon.

Fig. 3. As discussed and formally proven in Section V, this is a valid approximation because the RPC curve determined by this method is convex by construction.

The flowchart of the presented method for estimating the ADN's RPC areas for all time slots of the scheduling time horizon is sketched in Fig. 4.

\section{Linear Scenario-Based Robust Optimization PROBLEM FORMULATION}

\section{A. Mathematical Formulation}

This section describes the linear scenario-based robust optimization problem that determines a point on the RPC curve for a selected search direction. Assuming $\theta \neq 90^{\circ}, 270^{\circ}$, coefficients ap and $\mathrm{a}_{\mathrm{Q}}$ are respectively calculated based on (3) and (4). The associated point of the RPC curve is calculated by solving the following optimization problem:

$$
\min _{\psi} \mathrm{a}_{\mathrm{P}} R_{0^{\prime} t}^{\mathrm{P}}
$$

Subject to

$$
\begin{aligned}
& r_{0^{\prime} t s}^{\mathrm{Q}}=\mathrm{a}_{\mathrm{Q}} r_{0^{\prime} t s}^{\mathrm{P}} \\
& \mathrm{a}_{\mathrm{P}} r_{0^{\prime} t s}^{\mathrm{P}} \leq \mathrm{a}_{\mathrm{P}} R_{0^{\prime} t}^{\mathrm{P}} \leq 0 \\
& R_{0^{\prime} t}^{\mathrm{Q}}=\mathrm{a}_{\mathrm{Q}} R_{0^{\prime} t}^{\mathrm{P}} \\
& \Omega(\psi) \leq 0 \\
& \Gamma(\psi)=0
\end{aligned}
$$$$
\forall s \in \mathbb{S},
$$

$\forall s \in \mathbb{S}$

$\forall s \in \mathbb{S}$,

$\forall s \in \mathbb{S} .(10)$

where $\psi$ denotes the set of optimization variables of the problem:

$\psi=\left\{R_{0^{\prime} t}^{\mathrm{P}}, R_{0^{\prime} t}^{\mathrm{Q}}, r_{0^{\prime} t s}^{\mathrm{P}}, r_{0^{\prime} t s}^{\mathrm{Q}}, I_{l t s}^{\mathrm{real}}, I_{l t s}^{\mathrm{imag}}, V_{j t s}, r_{h t s}^{\mathrm{DG}, \mathrm{P}}, r_{h t s}^{\mathrm{DG}, \mathrm{Q}}, r_{k t s}^{\mathrm{SG}, \mathrm{Q}}\right\}$.

For ap $=-1$, the objective function (5) minimizes $-R_{0^{\prime} t}^{\mathrm{P}}$ or equivalently maximizes $R_{0^{\prime} t}^{\mathrm{P}}$, whereas, for ap $=+1$, it minimizes $R_{0^{\prime} t}^{\mathrm{P}}$. Constraint (6) specifies the search direction in the $R_{0^{\prime} t}^{\mathrm{P}}-R_{0^{\prime} t}^{\mathrm{Q}}$ plane, as shown in Fig. 3. Since the objective function (5) consists of the active power reserve capacity $R_{0^{\prime} t}^{\mathrm{P}}$ only, constraint (6) achieves to steer the search in the direction of the $R_{0^{\prime} t}^{\mathrm{Q}}$ axis also. In order to guarantee that the ADN can provide, in real-time operation, any amount of reserve corresponding to the points located in its RPC area in spite of uncertainties, this method computes robust estimates of the points on the RPC curve. In other words, $R_{0^{\prime} t}^{\mathrm{P}}$ and $R_{0^{\prime} t}^{\mathrm{Q}}$ (i.e., yellow squares in Fig. 3) are chosen as the most conservative $r_{0^{\prime} t s}^{\mathrm{P}}$ and $r_{0^{\prime} t s}^{\mathrm{Q}}$ (i.e., the innermost grey crosses in Fig. 3). This is performed by constraints (7) and (8). In (7), ap $=-1$ corresponds to the quadrants 1 and 4 (positive values of $r_{0^{\prime} t s}^{\mathrm{P}}$ and $R_{0^{\prime} t}^{\mathrm{P}}$ ), whereas ap $=+1$ corresponds to the quadrants 2 and 3 (negative values of $r_{0^{\prime} t s}^{\mathrm{P}}$ and $R_{0^{\prime} t}^{\mathrm{P}}$. Thanks to (6) and (7), the abovementioned conservative manner for $R_{0^{\prime} t}^{\mathrm{Q}}$ in particular can be modeled as constraint (8).

For search directions $\theta=90^{\circ}, 270^{\circ}$, the objective function and constraints (5)-(8) are modified as:

$$
\begin{array}{lc}
\min _{\psi} \mathrm{a}^{\prime} R_{0^{\prime} t}^{\mathrm{Q}} & \\
\text { Subject to } & \forall s \in \mathbb{S}, \\
r_{0^{\prime} t s}^{\mathrm{P}}=0 & \forall s \in \mathbb{S}, \\
\mathrm{a}^{\prime} r_{0^{\prime} t s}^{\mathrm{Q}} \leq \mathrm{a}^{\prime} R_{0^{\prime} t}^{\mathrm{Q}} \leq 0 & \forall s \in \mathbb{S}, \\
R_{0^{\prime} t}^{\mathrm{P}}=0 &
\end{array}
$$

in (5')-( $\left(8^{\prime}\right), \mathrm{a}^{\prime}$ is equal to -1 and +1 for $\theta=90^{\circ}$ and $\theta=$ $270^{\circ}$, respectively.

Expressions (9) and (10) are linear and model the operational constraints of the ADN and DERs, as described in Sections IV-B and IV-C.

The problem (5)-(10) is a linear scenario-based robust optimization problem that determines the point on the RPC curve associated to search direction $\theta$. In this problem, index $s$ corresponds to the scenarios modeling the forecast errors of loads $\left(\Delta \mathrm{P}_{i t s}^{\mathrm{D}}, \Delta \mathrm{Q}_{i t s}^{\mathrm{D}}\right)$ and stochastic generation $\left(\Delta \mathrm{P}_{k t s}^{\mathrm{SG}}\right)$. 


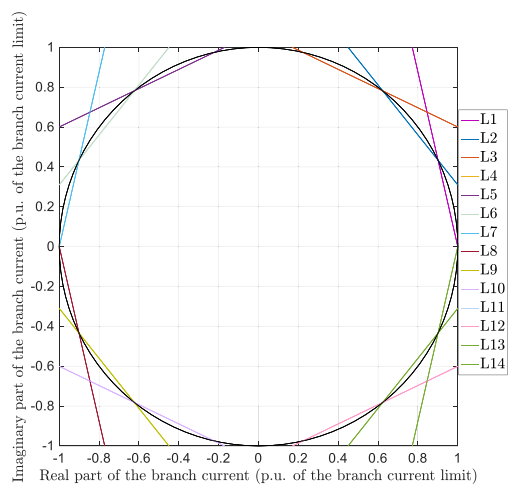

Fig. 5. Linearized branch current constraint.

In the following, the operational constraints of the ADN and DERs (9) and (10) are defined assuming that:

- dispatchable distributed generators (DDGs) can provide both active/reactive power reserves $\left(r_{h t s}^{\mathrm{DG}, \mathrm{P}}, r_{h t s}^{\mathrm{DG}, \mathrm{Q}}\right)$;

- SDGs are sources of active power uncertainties $\left(\Delta \mathrm{P}_{k t s}^{\mathrm{SG}}\right)$ and they can only provide reactive power reserves $\left(r_{k t s}^{\mathrm{SG}, \mathrm{Q}}\right)$;

- loads are sources of both active/reactive power uncertainties $\left(\Delta \mathrm{P}_{i t s}^{\mathrm{D}}, \Delta \mathrm{Q}_{i t s}^{\mathrm{D}}\right)$.

\section{B. Modeling the Technical Constraints of ADN}

The linearized load flow model proposed in [11] is used to derive expressions of:

- The active/reactive power reserve absorbed by the ADN from the upper-layer grid, at the ADN's connection point $\left(r_{0^{\prime} t s}^{\mathrm{P}}, r_{0^{\prime} t s}^{\mathrm{Q}}\right)$

- voltage magnitudes of all nodes of the $\operatorname{ADN}\left(V_{j t s}\right)$;

- current phasor of all branches of the $\operatorname{ADN}\left(I_{l t s}\right)$;

as a linear function of (voltage independent) nodal injections $\Delta P_{i t s}^{\mathrm{Net}}$ and $\Delta Q_{i t s}^{\mathrm{Net}}$.

The net deviation of the active/reactive power injection at node $i$ from its base case operating point (for the selected time slot $t$ ) during the scenario $s$ can be written as:

$$
\begin{aligned}
& \Delta P_{i t s}^{\mathrm{Net}}=-\Delta \mathrm{P}_{i t s}^{\mathrm{D}}+\sum_{h \in \mathbb{D} \mathbb{G}_{i}} r_{h t s}^{\mathrm{DG}, \mathrm{P}}+\sum_{k \in \mathbb{S G} \mathbb{G}_{i}} \Delta \mathrm{P}_{k t s}^{\mathrm{SG}} \forall i \in \mathbb{B}, \\
& \Delta Q_{i t s}^{\mathrm{Net}}=-\Delta \mathrm{Q}_{i t s}^{\mathrm{D}}+\sum_{h \in \mathbb{D} \mathbb{G}_{i}} r_{h t s}^{\mathrm{DG}, \mathrm{Q}}+\sum_{k \in \mathbb{S G}_{i}} r_{k t s}^{\mathrm{SG}, \mathrm{Q}} \forall i \in \mathbb{B} .
\end{aligned}
$$

The active/reactive power reserves absorbed by the ADN from the upper-layer grid are:

$$
\begin{aligned}
& r_{0^{\prime} t s}^{\mathrm{P}}=\mathbf{G}_{t}^{\mathrm{P} 0}+\sum_{i \in \mathbb{B}}\left(\mathbf{G}_{i t}^{\mathrm{PP}} \Delta P_{i t s}^{\mathrm{Net}}+\mathbf{G}_{i t}^{\mathrm{PQ}} \Delta Q_{i t s}^{\mathrm{Net}}\right) \forall s \in \mathbb{S}, \\
& r_{0^{\prime} t s}^{\mathrm{Q}}=\mathbf{G}_{t}^{\mathrm{Q} 0}+\sum_{\mathrm{i} \in \mathbb{B}}\left(\mathbf{G}_{i t}^{\mathrm{QP}} \Delta P_{i t s}^{\mathrm{Net}}+\mathbf{G}_{i t}^{\mathrm{QQ}} \Delta Q_{i t s}^{\mathrm{Net}}\right) \forall s \in \mathbb{S} .
\end{aligned}
$$

The maximum allowable current flow of branch $l$ can be expressed as the following nonlinear constraint:

$$
\left|I_{l t s}\right|^{2}=I_{l t s}^{\mathrm{real}^{2}}+I_{l t s}^{\mathrm{imag}^{2}} \leq \mathrm{I}_{l}^{\mathrm{Max}^{2}} \quad \forall l \in \mathbb{L}, \forall s \in \mathbb{S} .
$$

As represented in Fig. 5, the nonlinear constraint (15) is linearized based on a pre-defined number of linear boundaries

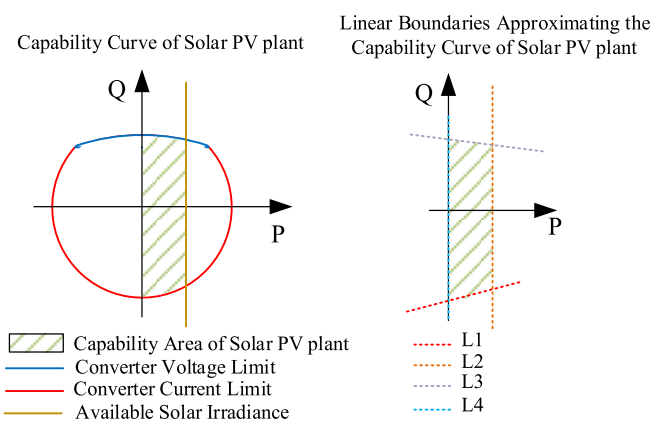

Fig. 6. Capability curve of a solar PV plant.

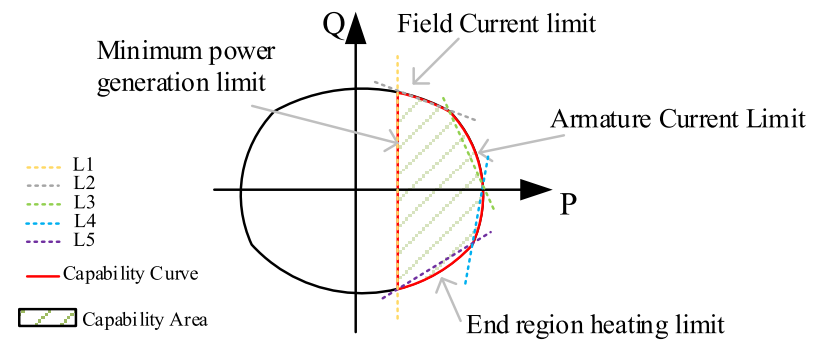

Fig. 7. Capability curve of a DDG.

approximating the real curve:

$\mathbf{F}_{f l}^{\mathrm{real}} I_{l t s}^{\mathrm{real}}+\mathbf{F}_{f l}^{\mathrm{imag}} I_{l t s}^{\mathrm{imag}} \leq \mathbf{F}_{f l}^{0} \quad \forall l \in \mathbb{L}, \quad \forall f \in \mathrm{F}_{l}, \quad \forall s \in \mathbb{S}$,

where the real and imaginary part of the current phasor of branch $l^{1}$ are expressed as a linear function of the nodal active/reactive power deviations:

$$
\begin{array}{r}
I_{l t s}^{\text {real }}=\mathbf{H}_{l t}^{0, \text { real }}+\sum_{i \in \mathbb{B}}\left(\mathbf{H}_{l i t}^{\mathrm{P}, \text { real }} \Delta P_{i t s}^{\mathrm{Net}}+\mathbf{H}_{l i t}^{\mathrm{Q}, \text { real }} \Delta Q_{i t s}^{\mathrm{Net}}\right) \\
\forall l \in \mathbb{L}, \quad \forall s \in \mathbb{S}, \\
I_{l t s}^{\mathrm{imag}}=\mathbf{H}_{l t}^{0, \mathrm{imag}}+\sum_{i \in \mathbb{B}}\left(\mathbf{H}_{l i t}^{\mathrm{P}, \mathrm{imag}} \Delta P_{i t s}^{\mathrm{Net}}+\mathbf{H}_{l i t}^{\mathrm{Q}, i m a g} \Delta \mathrm{Q}_{i t s}^{\mathrm{Net}}\right) \\
\forall l \in \mathbb{L}, \quad \forall s \in \mathbb{S} .
\end{array}
$$

The voltage magnitude of node $j$ can be expressed as a linear function of the nodal active/reactive power deviations. Consequently, the nodal voltage magnitude limits can be linearly modeled as:

$$
\begin{array}{r}
\mathrm{V}_{j}^{\mathrm{Min}} \leq V_{j t s}=\mathbf{E}_{j t}^{0}+\sum_{i \in \mathbb{B}}\left(\mathbf{E}_{j i t}^{\mathrm{P}} \Delta P_{i t s}^{\mathrm{Net}}+\mathbf{E}_{j i t}^{\mathrm{Q}} \Delta Q_{i t s}^{\mathrm{Net}}\right) \leq \mathrm{V}_{j}^{\mathrm{Max}} \\
\forall j \in \mathbb{B}, \forall s \in \mathbb{S} .
\end{array}
$$

It is worth noting that the expressions (11)-(19) are applicable to both meshed and radial grids by virtue of the linearized grid model. Their coefficients are functions of the grid admittance matrix, nodal active/reactive power injections for the base case operating point and the voltage magnitude at node 0 ' (slack node) [11].

\footnotetext{
${ }^{1}$ The maximum current flow limit of branch $l$ is modeled for its both sending and receiving ends.
} 


\section{Modeling the Capability Limits of DERs}

The power capability limits of DERs (typically nonlinear), are linearized by using a pre-defined number of linear boundaries. For instance, Fig. 6 shows the capability curve of a solar PV plant from [12], and its linearized version. Similarly, Fig. 7 shows the capability curve of a DDG from [13], like a gas turbine.

Based on the linearized models of the capability limits of DDGs and SDGs, the operational constraints of SDG $k$ and DDG $h$ are as:

$$
\begin{aligned}
\mathbf{M}_{n k t s}^{0}+\mathbf{M}_{n k t s}^{\mathrm{P}} \Delta \mathrm{P}_{k t s}^{\mathrm{SG}}+\mathbf{M}_{n k t s}^{\mathrm{Q}} r_{k t s}^{\mathrm{SG}, \mathrm{Q}} \leq 0 \\
\forall n \in \mathbb{C}_{k}, \quad \forall k \in \mathbb{S} G, \forall s \in \mathbb{S}, \\
\mathbf{K}_{n h t}^{0}+\mathbf{K}_{n h}^{\mathrm{P}} r_{h t s}^{\mathrm{DG}, \mathrm{P}}+\mathbf{K}_{n h}^{\mathrm{Q}} r_{h t s}^{\mathrm{DG}, \mathrm{Q}} \leq 0 \\
\forall n \in \mathbb{C}_{h}, \quad \forall h \in \mathbb{D} \mathbb{G}, \quad \forall s \in \mathbb{S} .
\end{aligned}
$$

\section{ON THE CONVEXITY OF THE RPC AREA OF ADN}

Relying on the linearized model of the ADN constraints presented in Section IV-B, it is formally proven in Appendix A that the RPC area is convex. Therefore, the following theorems, are introduced.

Theorem 1: Assuming that the capability limits of all DERs of the ADN are convex areas $^{2}$ and that the linearized grid model (16)-(19) holds, the RPC area of ADN for a given scenario is a convex area.

Theorem 2: The RPC area of the ADN obtained by considering all scenarios is convex in the $R_{0^{\prime} t}^{\mathrm{P}}-R_{0^{\prime} t}^{\mathrm{Q}}$ plane.

Based on Theorems 1 and 2, the main results of this section is now presented. As the RPC area is convex, it is possible to conclude that linearly interpolating the points as done in step 3, achieves a conservative feasible approximation of the RPC curve.

\section{Determining the Number of Scenarios}

This section aims at determining the number of scenarios belonging to $\mathbb{S}$, i.e., $\mathrm{N}_{\mathrm{s}}$, which should be taken into account in the scenario-based robust optimization problem (5)-(10), in order to guarantee that the achieved optimal solution (i.e., $R_{0^{\prime} t}^{\mathrm{P}}, R_{0^{\prime} t}^{\mathrm{Q}}$ ) satisfies all the grid constraints considering all possible realizations of the uncertain parameters (i.e., loads $\Delta \mathrm{P}_{i t s}^{\mathrm{D}}, \Delta \mathrm{Q}_{i t s}^{\mathrm{D}}$ and stochastic generation $\left.\Delta \mathrm{P}_{i t s}^{\mathrm{SSG}}\right)$ with an expected probability larger or equal than $1-\varepsilon$ and with a confidence level of $1-\beta$. Relying on the analysis of [15], it is proven in Appendix B that the above-mentioned target is achieved provided that:

$$
\mathrm{N}_{s} \geq \frac{2}{\varepsilon} \ln \frac{1}{\beta}+2\left(2 \mathrm{~N}^{\mathrm{DG}}+\mathrm{N}^{\mathrm{SG}}\right)+\frac{2\left(2 \mathrm{~N}^{\mathrm{DG}}+\mathrm{N}^{\mathrm{SG}}\right)}{\varepsilon} \ln \frac{2}{\varepsilon},
$$

where $\mathrm{N}^{\mathrm{DG}}$ and $\mathrm{N}^{\mathrm{SG}}$ are the number of DDGs and SDGs installed in the ADN, respectively.

\footnotetext{
${ }^{2}$ Capability curves of DERs are typically convex, see for instance [12]-[14].
}

TABLE I

CHARACTERISTICS OF SDGS

\begin{tabular}{cc||cc}
\hline \multicolumn{2}{c||}{ Solar PV SDGs } & \multicolumn{2}{c}{ Wind SDGs } \\
\hline $\begin{array}{c}\text { Connected to } \\
\text { node }\end{array}$ & $\begin{array}{c}\text { Nominal power } \\
(\mathrm{kVA})\end{array}$ & $\begin{array}{c}\text { Connected to } \\
\text { node }\end{array}$ & $\begin{array}{c}\text { Nominal power } \\
(\mathrm{kVA})\end{array}$ \\
\hline \hline 5,22 & 300 & 11 & 300 \\
\hline 14,20 & 350 & 16,26 & 450 \\
\hline
\end{tabular}

TABLE II

CHARACTERISTICS OF DDGS

\begin{tabular}{ccc}
\hline Connected to node & $\begin{array}{c}\text { Nominal power } \\
\text { (kVA) }\end{array}$ & $\begin{array}{c}\text { Minimum active power } \\
\text { generation limit (kW) }\end{array}$ \\
\hline \hline 3,9 & 500 & 10 \\
\hline 17,31 & 700 & 20 \\
\hline
\end{tabular}

\section{Case Study and Results}

\section{A. Case Study}

The modified IEEE 33-bus distribution test system [16], shown in Fig. 2, is utilized as the case study to derive the RPC area. The modification refers to the presence of SDGs, composed by solar PV and wind generators, and DDGs. The characteristics of SDGs and DDGs are given in Table I and Table II, respectively. In line with the reserve allocation procedure introduced in Section II, it is considered that:

- the scheduling time horizon is 24 hours and refers to the next day;

- the next day consists of 24 time slots, each with duration of 1-hour;

- the RPC area of an ADN for each time slot of the next day is estimated one hour before the beginning of the next day.

The solar PV and load profiles, for 24 hours of study, are real measurements from a monitored primary high-to-medium voltage substation in the south of Switzerland. The wind profiles are from [17]. The loads are assumed voltage-independent and with power factor equal to 0.95. Injections of both SDGs and DDGs are also assumed voltage-independent. Statutory minimum/maximum voltage limits are chosen as $0.95 \mathrm{pu}$ and $1.05 \mathrm{pu}$.

It is assumed that the day-ahead forecast errors of the nodal active power consumption/generation are independent and identically distributed. The active power forecast error of each load is sampled from a normal distribution with 0 mean and such that the standard deviation of the total load forecast error is $3 \%$ of the total load of the ADN, as in [18]. The deviation of active power generation of each SDG from its forecasted value is sampled from a normal distribution with 0 mean and such that the standard deviation of the whole system stochastic generation forecast error is $7 \%$ of the total system stochastic generation forecast, as in [19]. This approach is used to model the uncertainties. The number of scenarios, $\mathrm{N}_{\mathrm{s}}$, is determined based on the approach introduced in Section VI. To this end, the upper bound of the probability of constraint violation by the optimal solution at a random realization of the uncertainties, $\varepsilon$, is fixed equal to $4 \%$ with confidence level, $1-\beta$, equal to $99.99 \%$. It 


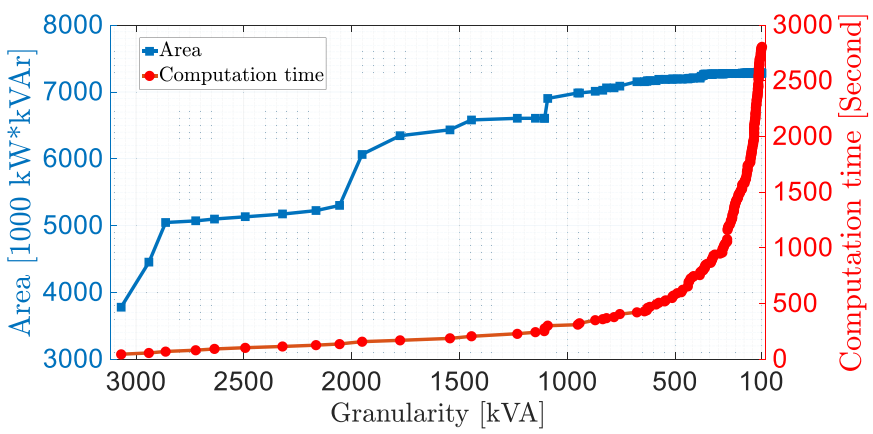

Fig. 8. Impact of the defined granularity on the precision of the estimated RPC and its computation time $\left(\mathrm{N}_{\mathrm{s}}=3000\right)$.

should be noted that $\varepsilon$ also refers to the violation of any single constraint. Considering expression (22) along with $\mathrm{N}^{\mathrm{DG}}=4$ and $\mathrm{N}^{\mathrm{SG}}=7$, the number of scenarios should be 2904 . Accordingly, $\mathrm{N}_{\mathrm{s}}$ is slightly overestimated and defined equal to 3000 .

It should be noted that selecting an algorithm for solving the developed linear optimization problem (5)-(10) is mandatory but it is not crucial. In fact, the main contribution and novelty of our paper is to introduce a linear (convex) formulation with low complexity for estimating the RPC area of ADNs while considering the uncertainties. It is here decided to solve the problem using linear programming solver of GUROBI [20]. Thus, the problem is modelled by using YALMIP-MATLAB [21] and solved with GUROBI [20] on a Windows based system with a $2.8 \mathrm{GHz}$ Xeon CPU and 32 GB of RAM. However, the heuristic algorithms described in [22] and [23] are also applicable for solving the problem.

\section{B. Impact of the Granularity on the Estimated RPC Area}

The impact of the granularity on the precision of the estimated RPC area and on the computation time is investigated for several values of the granularity. Fig. 8 shows the surface the RPC area and computation time for the first time slot of the day as a function of the granularity. It shows that, by increasing the granularity, the precision of the estimated RPC area improves at the cost of a higher computation time. However, when above $400 \mathrm{kVA}$, the precision of the estimated RPC does not change considerably, while the computation time drastically increases. Therefore, in the following analysis, $400 \mathrm{kVA}$ is retained as the value of granularity as it achieves a reasonable trade-off between degree of approximation and computational time. The estimated RPC areas for granularity values of $400 \mathrm{kVA}$ and $100 \mathrm{kVA}$ are shown in Fig. 9. As it can be seen, the two RPC areas are similar, thus denoting that, in this case, the lower granularity achieves a good degree of approximation.

\section{Impact of the Chosen Number of Scenarios on the Estimated RPC Area}

The impact of the number of scenarios, $\mathrm{N}_{\mathrm{s}}$, on the precision of the estimated RPC area and on the computation time is investigated by choosing several values of $\mathrm{N}_{\mathrm{s}}$. Fig. 10 shows

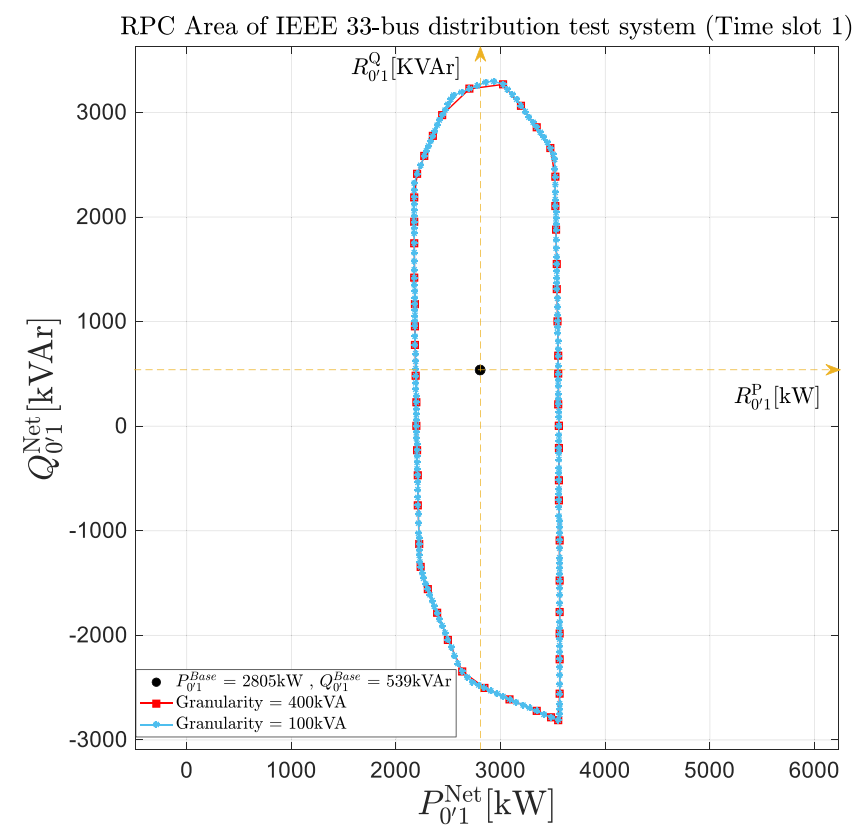

Fig. 9. Estimated RPC area for the maximum granularity equal to $100 \mathrm{kVA}$ and $400 \mathrm{kVA}$.

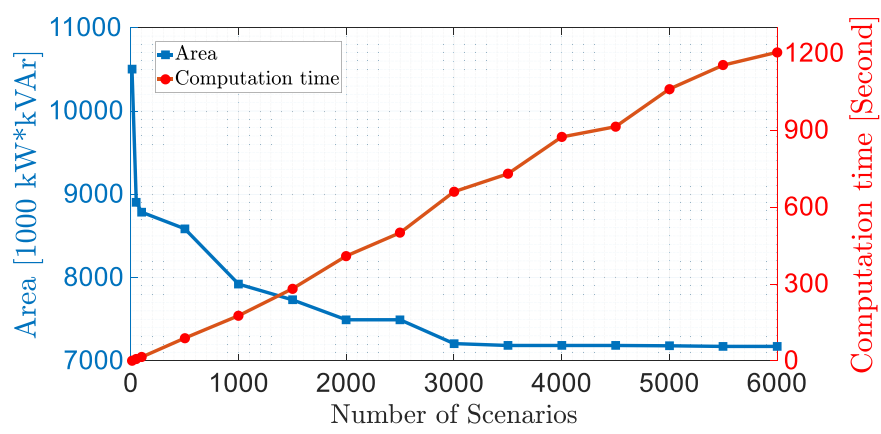

Fig. 10. Impact of the chosen number of scenarios on the precision of the estimated RPC, and its computation time (granularity $=400 \mathrm{KVA}$ ).

the surface of RPC area and computation time for the first time slot of the day as a function of $\mathrm{N}_{\mathrm{s}}$. It corroborates that:

- estimating the RPC area considering only a single scenario, i.e., neglecting uncertainties, leads to a RPC area which is significantly far from the one that considers uncertainties;

- by increasing $\mathrm{N}_{\mathrm{s}}$, the precision of the estimated RPC area improves at the cost of a higher computation time. However, above 3000, the precision of the estimated RPC does not change significantly, whereas the computation time drastically increases.

\section{Evolution Over Time of the Estimated RPC Areas}

The RPC areas of the ADN are estimated for all 24 time slots of study and are shown in Fig. 11. In this figure, the $\mathrm{RPC}$ areas are represented in $R_{0^{\prime} t}^{\mathrm{P}}-R_{0^{\prime} t}^{\mathrm{Q}}$ plane instead of $P_{0^{\prime} t}^{\mathrm{Net}}-Q_{0^{\prime} t}^{\mathrm{Net}}$ to better highlight the evolution of the RPC areas over the period of study. As it can be seen, the RPC areas of all time intervals have different shapes, denoting that integrating forecasting scenarios is critical to achieve 


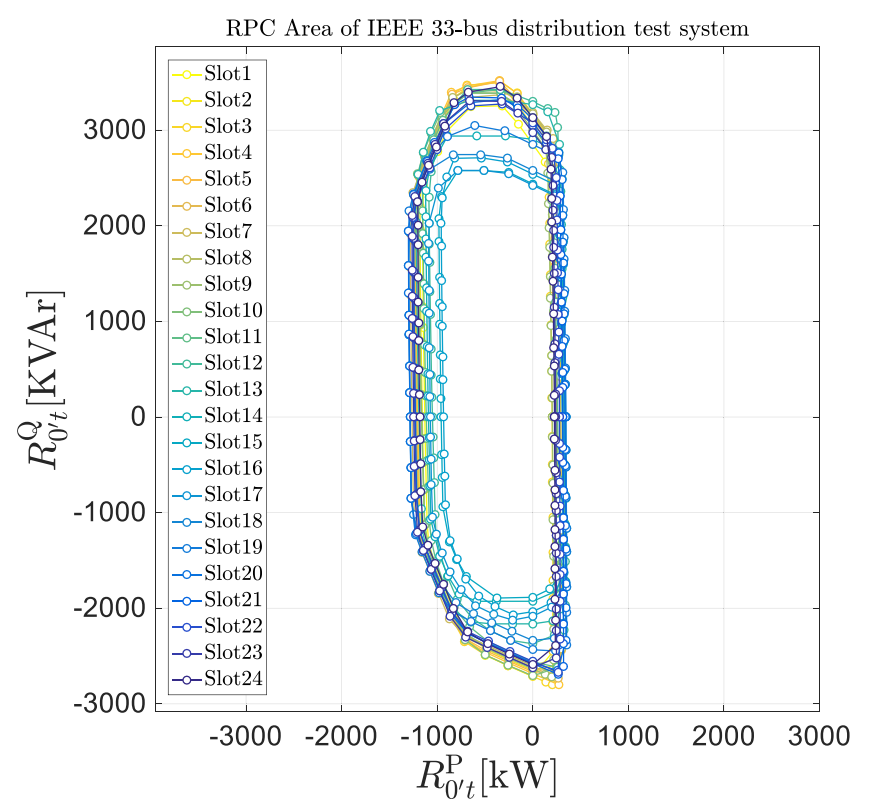

Fig. 11. The superimposed RPC area of all 24 time slots of the study.

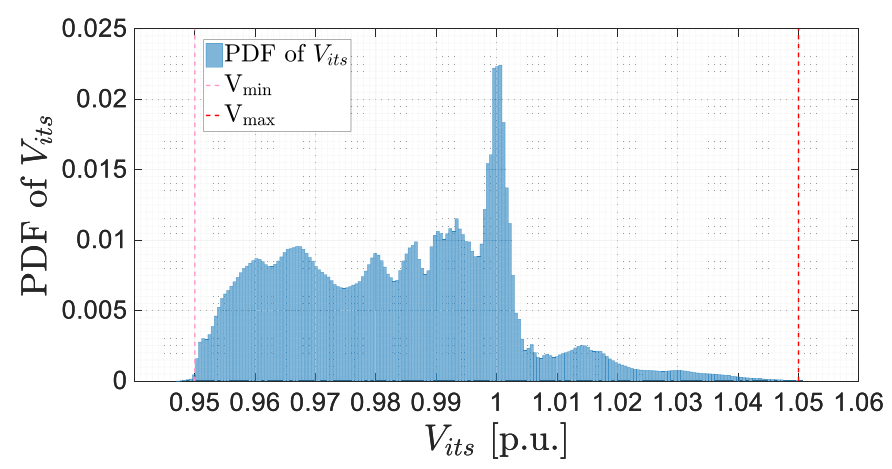

Fig. 12. Probability density function of the voltage magnitudes of all buses (for the vertexes forming RPC curve) extracted from the AC power flow results.

an accurate representation of the dynamic capacity of ADNs for reserve provision. As expected, the RPC areas shown in Fig. 11 are convex, therefore they can be embedded efficiently in existing convex optimization tools [24] used by operators for optimal allocation of power reserves without impacting on their tractability. The RPC area of each time slot is estimated in less than 500 seconds.

\section{E. On the Feasibility of the Estimated RPC Area}

The proposed method for estimating the RPC area models grid constraints using a linearized power flow model, thus, the estimated RPC area may overestimate the real one. In other words, some points belonging to the estimated RPC area may actually be infeasible operating points. In this respect, since the feasibility of all the estimated RPC area points cannot be explicitly investigated, only those suspected to be infeasible are investigated. The selected points belong to the estimated RPC curve. Actually, we consider only the vertexes that permitted to build that curve. According to our modeling, for each of these vertexes, one or more grid/DERs constraints are activated meaning that vertex is supposed to be close to the

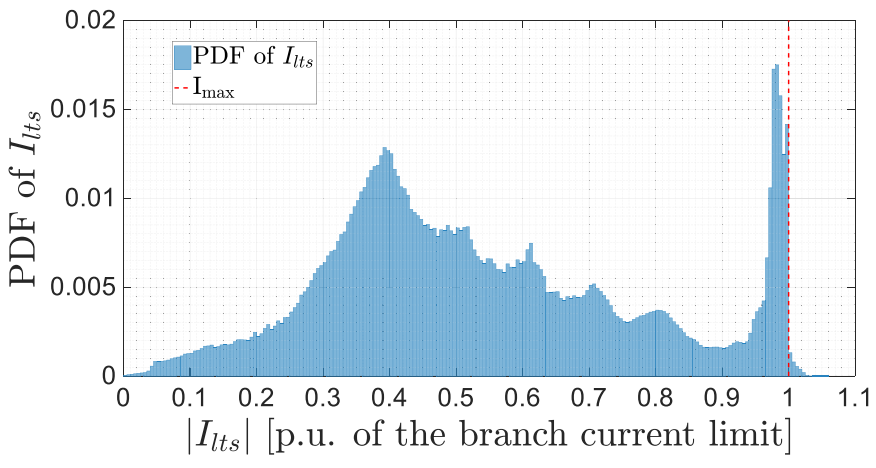

Fig. 13. Probability density function of the current magnitude of all branches (for the vertexes forming RPC curve) extracted from the AC power flow results.

infeasibility region. This is assumed to be a pertinent choice even if it could appear not sufficient to decide on the feasibility of all the estimated RPC area. A vertex of the RPC curve is considered feasible if the corresponding AC power flow result satisfies all grid constraints. Considering all 24 time slots and all vertexes of the RPC curves (as shown in Fig. 11), the probability density functions (PDFs) of the voltage magnitude of all nodes and current magnitude of all branches (extracted from the AC power flow results) can be built as shown in Fig. 12 and Fig. 13, respectively. Fig. 12 and Fig. 13 corroborate that a single voltage and current constraint were violated in less than $0.057 \%$ and $0.3 \%$ of the cases, respectively.

\section{CONCLUSION}

This paper proposes a method to estimate the RPC area of an ADN for a desired time horizon while considering forecast errors, and operational constraints of the grid (i.e., nodal voltages and line currents, modeled with a linearized power flow model) and of DERs. The method consists in solving, for a certain search direction in the $R_{0^{\prime} t}^{\mathrm{P}}-R_{0^{\prime} t}^{\mathrm{Q}}$ plane, an optimization problem to estimate the maximum active and reactive power reserves that the $\mathrm{ADN}$ can provide in that direction, allowing to establish a point of the RPC curve. The procedure is initially applied to the four main search directions (i.e., 0, 90, 180 , and 270 degrees), and then repeated for angle submultiples until the distance between each consecutive couple of estimated points of the RPC curve is less than a pre-defined granularity. The identified points are linearly interpolated and used to approximate the RPC area. It is proven that, assuming convex capabilities curves of distributed energy resources and a linearized power flow model, the estimated RPC area is feasible and convex. The optimization problem relies on a linearized grid model to represent grid constraints and forecasts scenarios to model uncertainties of loads and renewable generation. This tractable linear robust formulation achieves to estimate the RPC area efficiently while hedging against uncertainties and accounting for typical operational constraints of ADNs.

The modified IEEE 33-bus test system is used to illustrate the effectiveness and capability of the proposed method. A 24hour time horizon with 24 time slots is chosen to estimate the RPC area. The results show that, for the considered case 
study, the method is able to precisely estimate the RPC area for the whole scheduling horizon in less than 600 seconds when selecting $400 \mathrm{kVA}$ as the granularity level. Also, they show that the accuracy of the estimated RPC area, in the considered case study, does not improve when increasing the granularity to less than $400 \mathrm{kVA}$. As expected from the formulation, all estimated RPC areas are convex, thus they can be conveniently embedded in upper-layer decision problems without impacting tractability and convexity.

\section{APPENDIX A}

Proof of Theorem 1: Let $\boldsymbol{X}_{t s}=\left[\Delta P_{1 t s}^{\mathrm{Net}}, \ldots, \Delta\right.$ $\left.P_{\mathrm{N}_{\mathbb{B}} t s}^{\mathrm{Net}}, \Delta Q_{1 t s}^{\mathrm{Net}}, \ldots, \Delta Q_{\mathrm{N}_{\mathbb{B}} t s}^{\mathrm{Net}}\right]^{\mathrm{T}}$ denote the vector of the operating point of the ADN collecting the deviations of the nodal active/reactive power injections. For the given scenario $s$, i.e., given operating point $\boldsymbol{X}_{t s}$, the corresponding point $\left[r_{0^{\prime} t s}^{\mathrm{P}}, r_{0^{\prime} t s}^{\mathrm{Q}}\right]^{\mathrm{T}}$ in the $R_{0^{\prime} t}^{\mathrm{P}}-R_{0^{\prime} t}^{\mathrm{Q}}$ plane can be calculated using (13) and (14). Contrariwise, for the given point $\left[r_{0^{\prime} t s}^{\mathrm{P}}, r_{0^{\prime} t s}^{\mathrm{Q}}\right]^{\mathrm{T}}$, the corresponding operating point $\boldsymbol{X}_{t s}$ can be calculated as:

$$
\begin{aligned}
& \Delta P_{i t s}^{\mathrm{Net}}=\mathbf{G}_{i t}^{\prime \mathrm{P} 0}+\sum_{i \in \mathbb{B}}\left(\mathbf{G}_{i t}^{\prime \mathrm{PP}} r_{0^{\prime} t s}^{\mathrm{P}}+\mathbf{G}_{i t}^{\prime \mathrm{PQ}} r_{0^{\prime} t s}^{\mathrm{Q}}\right) \forall i \in \mathbb{B}, \\
& \Delta Q_{i t s}^{\mathrm{Net}}=\mathbf{G}_{i t}^{\prime \mathrm{Q} 0}+\sum_{\mathrm{i} \in \mathbb{B}}\left(\mathbf{G}_{i t}^{\mathrm{QP}} r_{0^{\prime} t s}^{\mathrm{P}}+\mathbf{G}_{i t}^{\prime \mathrm{QQ}} r_{0^{\prime} t s}^{\mathrm{Q}}\right) \forall i \in \mathbb{B} .
\end{aligned}
$$

Operating point $\boldsymbol{X}_{t s}$ is said feasible if it satisfies the capability limits of all DERs and the operational constraints of the ADN in (16) and (19).

Based on the above-mentioned notations and definitions along with the assumptions of theorem, the goal here is to show that for each two given points 1 and 2 belonging to the RPC area of scenario $s$, all points located on the line connecting them to each other also belong to that RPC area. Each point located on this line like point 3 can be mathematically represented as (hereafter superscripts 1,2 and 3 denote the index of points):

$$
\left[\begin{array}{cc}
r_{0^{\prime}}^{\mathrm{P}} & 3 \\
0^{\prime} t s & 3 \\
r_{0^{\prime} t s}^{\mathrm{Q}} & 3
\end{array}\right]=(1-\alpha)\left[\begin{array}{cc}
r_{0^{\prime}}^{\mathrm{P}} & 1 \\
0^{2} t s & \\
r_{0^{\prime} t s}^{\mathrm{Q}} & 1
\end{array}\right]+\alpha\left[\begin{array}{cc}
r_{0^{\prime} t s}^{\mathrm{P}} & 2 \\
r_{0^{\prime} t s}^{\mathrm{Q}} & 2
\end{array}\right],
$$

where $\alpha$ is a constant between 0 and 1 .

Combination of (A1.1)-(A1.3) yields:

$$
\boldsymbol{X}_{t s}^{3}=(1-\alpha) \boldsymbol{X}_{t s}^{1}+\alpha \mathrm{X}_{t s}^{2} .
$$

Note that points 1 and 2 belong to the RPC area and they are feasible, thus, it can be inferred that:

1- Points $\boldsymbol{X}_{t s}^{1}$ and $\boldsymbol{X}_{t s}^{2}$ satisfy the capability limits of all DERs limits.

2- Points $\boldsymbol{X}_{t s}^{1}$ and $\boldsymbol{X}_{t s}^{2}$ satisfy the nodal voltage constraint of ADN. Based on (19), it can be mathematically expressed for each node like $j$ as:

$$
\begin{aligned}
& \mathrm{V}_{j}^{\mathrm{Min}} \leq \mathbf{E}_{j t}^{0}+\sum_{i \in \mathbb{B}}\left(\mathbf{E}_{j i t}^{\mathrm{P}} \Delta P_{i t s}^{\mathrm{Net}^{1}}+\mathbf{E}_{j i t}^{\mathrm{Q}} \Delta Q_{i t s}^{\mathrm{Net}^{1}}\right) \leq \mathrm{V}_{j}^{\mathrm{Max}}, \\
& \mathrm{V}_{j}^{\mathrm{Min}} \leq \mathbf{E}_{j t}^{0}+\sum_{i \in \mathbb{B}}\left(\mathbf{E}_{j i t}^{\mathrm{P}} \Delta P_{i t s}^{\mathrm{Net}^{2}}+\mathbf{E}_{j i t}^{\mathrm{Q}} \Delta Q_{i t s}^{\mathrm{Net}^{2}}\right) \leq \mathrm{V}_{j}^{\mathrm{Max}} .
\end{aligned}
$$

3- Points $\boldsymbol{X}_{t s}^{1}$ and $\boldsymbol{X}_{t s}^{2}$ satisfy the maximum current flow limits of all branches. Based on (16), it can be mathematically expressed for each branch like $l$ as:

$$
\begin{aligned}
& \mathbf{F}_{f l}^{\mathrm{real}} I_{l t s}^{\mathrm{real}^{1}}+\mathbf{F}_{f l}^{\mathrm{imag}} I_{l t s}^{\mathrm{imag}^{1}} \leq \mathbf{F}_{f l}^{0} \forall f \in \mathrm{F}_{l}, \\
& \mathbf{F}_{f l}^{\mathrm{real}} I_{l t s}^{\mathrm{real}^{2}}+\mathbf{F}_{f l}^{\mathrm{imag}} I_{l t s}^{\mathrm{imag}^{2}} \leq \mathbf{F}_{f l}^{0} \forall f \in \mathrm{F}_{l} .
\end{aligned}
$$

1) Proof of $\boldsymbol{X}_{t s}^{\mathbf{3}}$ Satisfies the Capability Limits of DERs: Both $\boldsymbol{X}_{t s}^{1}$ and $\boldsymbol{X}_{t s}^{2}$ satisfy the capability limits of all DERs. Since capability limits of DERs are assumed to be convex areas, thus any point like $\boldsymbol{X}_{t s}^{3}$ defined based on (A1.4) satisfies these capability limits.

2) Proof of $\boldsymbol{X}_{t s}^{\mathbf{3}}$ Satisfies the Nodal Voltage Constraint of $A D N$ : It is shown that for the given points $\boldsymbol{X}_{t s}^{1}$ and $\boldsymbol{X}_{t s}^{2}$ respectively satisfying (A1.5) and (A1.6), any point like $\boldsymbol{X}_{t s}^{3}$ defined based on (A1.4) satisfies (19). To this end, first (A1.5) and (A1.6) are respectively multiplied by $(1-\alpha)$ and $\alpha$. Then they are added to each other and composed with (A1.4) yielding:

$$
\mathrm{V}_{j}^{\mathrm{Min}} \leq \mathbf{E}_{j t}^{0}+\sum_{i \in \mathbb{B}}\left(\mathbf{E}_{j i t}^{\mathrm{P}} \Delta P_{i t s}^{\mathrm{Net}^{3}}+\mathbf{E}_{j i t}^{\mathrm{Q}} \Delta Q_{i t s}^{\mathrm{Net}^{3}}\right) \leq \mathrm{V}_{j}^{\mathrm{Max}}
$$

Expression (A1.9) demonstrates that $\mathbf{X}_{t s}{ }^{3}$ satisfies the nodal voltage constraint of ADN.

3) Proof of $\boldsymbol{X}_{t \mathbf{s}}^{\mathbf{3}}$ Satisfies the Maximum Current Flow Limits of All Branches: Considering the given points $\mathbf{X}_{t s}^{1}$ and $\mathbf{X}_{t s}{ }^{2}$ respectively satisfying (A1.7) and (A1.8), it is shown that any point like $\mathbf{X}_{t s}{ }^{3}$ defined based on (A1.4) satisfies (16). To this end, first (A1.7) and (A1.8) are respectively multiplied by $(1-\alpha)$ and $\alpha$. Then they are added to each and composed with (17), (18) and (A1.4) yielding:

$$
\mathbf{F}_{f l}^{\mathrm{real}} I_{l t s}^{\mathrm{real}^{3}}+\mathbf{F}_{f l}^{\mathrm{imag}} I_{l t s}^{\mathrm{imag}^{3}} \leq \mathbf{F}_{f l}^{0} \quad \forall f \in \mathbb{F}_{l} .
$$

Expression (A1.10) demonstrates that $\boldsymbol{X}_{t s}^{3}$ satisfies the maximum current flow limits of all branches.

Based on the above-mentioned proofs, it is trivial that $\mathbf{X}_{t s}{ }^{3}$ is a feasible points and consequently $\left[r_{0^{\prime} t s}^{\mathrm{P}}{ }^{3}, r_{0^{\prime} t s}^{\mathrm{Q}}{ }^{3}\right]^{\mathrm{T}}$ belongs to the RPC area of ADN.

Proof of Theorem 2: The method introduced in III defines the boundary of the RPC area, in each direction $\theta$ (as shown in Fig. 3), as the minimum amount of reserve that ADN can provide over all scenarios. It can be mathematically expressed as:

$$
A_{\mathbb{S}}^{\mathrm{RPC}}=\bigcap_{s \in \mathbb{S}} A_{s}^{\mathrm{RPC}},
$$

where $A_{\mathbb{S}}^{\mathrm{RPC}}$ indicate the RPC area obtained by considering all scenarios belonging to $\mathbb{S}, A_{s}^{\mathrm{RPC}}$ indicate the RPC area obtained by considering single scenario $s$ and Operator $\cap$ calculates the intersection of all areas.

On the one hand, Theorem 1 proved that $A_{s}^{\mathrm{RPC}}$ is convex for each scenario $s$. On the other hand, the intersection of a number of convex areas returns a convex area [25]. Thus, it is trivial that $A_{\mathbb{S}}^{\mathrm{RPC}}$ is convex. 


\section{APPENDiX B}

For the sake of brevity, let us consider an abstract representation of a linear robust optimization problem. To this end, $\mathcal{R}$ is used for the set of real numbers; $\mathrm{N}_{\mathrm{v}}$ for the number of optimization variables; vector $x \in \mathcal{R}^{\mathrm{N}_{\mathrm{v}}}$ for the optimization variables; linear function $\mathrm{f}(\boldsymbol{x}) \in \mathcal{R}$ for the optimization objective function; $\mathrm{N}_{\mathrm{u}}$ for the number of uncertain parameters; vector $\mathbf{u} \in \mathcal{R}^{\mathrm{N}_{\mathrm{u}}}$ for the uncertain parameters; vector $u^{\max } \in \mathcal{R}^{\mathrm{N}_{u}}$ for the upper-limit of $\mathbf{u}$; vector $u^{\min } \in \mathcal{R}^{\mathrm{N}_{\mathrm{u}}}$ for the lower-limit of $\mathbf{u} ; \mathrm{N}_{\mathrm{c}}$ for the number of optimization constraints; linear vector-function $\mathcal{C}(\boldsymbol{x}, u) \in \mathcal{R}^{\mathrm{N}_{\mathrm{c}}}$ for the optimization constraints. Now, a linear robust optimization problem can be mathematically stated as:

$$
\min _{x} \mathrm{f}(\boldsymbol{x})
$$

\section{subject to}

$$
\mathcal{C}(\boldsymbol{x}, \boldsymbol{u}) \leq 0 \quad \forall \mathbf{u}^{\min } \leq \mathbf{u} \leq \mathbf{u}^{\max } .
$$

Due to the presence of $\mathbf{u}$ in (A2.2), problem (A2.1)-(A2.2) encompasses an infinite number of constraints, thus, it is a semi-infinite optimization problem for which finding the solution is a formidable task [15], [26]. A computationally feasible technique to solve this robust optimization problem is to convert it to a scenario-based robust optimization problem. To this purpose, let first slightly relax its constraints and convert it to a chance-constrained optimization problem as:

$\min _{x} \mathrm{f}(\boldsymbol{x})$

subject to

Probability $\{\mathcal{C}(\boldsymbol{x}, \mathbf{u})>0\} \leq \varepsilon \quad \forall \mathbf{u}^{\min } \leq \mathbf{u} \leq \mathbf{u}^{\max }$,

where $0 \leq \varepsilon \leq 1$ indicates the allowable probability of the constraints violation. In this problem, it is accepted that the optimal solution violates constraints with expected probability of less than $\varepsilon$ for all possible realizations of $\mathbf{u}$. It is obvious that the optimal solution of the chance-constrained problem outperforms the optimal solution of its robust counterpart. However, it is notable that the chance-constrained problem (A2.3)-(A2.4) gets closer to its robust counterpart (A2.1)-(A2.2) for decreasing $\varepsilon$. This chance-constrained optimization problem again similar to its robust counterpart is intractable. In order to solve this problem, [15] devised the scenario-based robust optimization problem as:

$$
\min _{x} \mathrm{f}(\boldsymbol{x})
$$

Subject to

$$
\mathcal{C}\left(\boldsymbol{x}, \mathbf{u}_{s}\right) \leq 0 \quad \forall s \in \mathbb{S},
$$

where $u_{s}$ is one realization of $\mathbf{u}$ and $\mathbf{u}^{\min } \leq \mathbf{u}_{s} \leq \mathbf{u}^{\max }$. The optimal solution of the scenario-based robust optimization must only satisfy the constraints for a limited number, $\mathrm{N}_{\mathrm{s}}$, of scenarios (i.e., realization of uncertainties). Reference [15], mathematically proved that the optimal solution of the scenario-based robust optimization problem (A2.5)-(A2.6) is also an optimal solution of the chance-constrained problem (A2.3)-(A2.4) with feasibility level $1-\varepsilon$ and confidence interval $1-\beta$, provided that:

$$
\mathrm{N}_{s} \geq \frac{2}{\varepsilon} \ln \frac{1}{\beta}+2 \mathrm{~N}_{\mathrm{v}}+\frac{2 \mathrm{~N}_{\mathrm{v}}}{\varepsilon} \ln \frac{2}{\varepsilon},
$$

it illustrates that if the problem (A2.5)-(A2.6) is solved for the finite number $\mathrm{N}_{\mathrm{S}}$ of scenarios, then, the achieved optimal solution satisfies all constraints for most of the other unseen scenarios. In other words, the explicit satisfaction of $\mathrm{N}_{\mathrm{s}}$ scenarios automatically generalizes the satisfaction of other scenarios. Therefore, in order to solve the robust optimization problem (A2.1)-(A2.2) with feasibility level $1-\varepsilon$ and confidence interval $1-\beta$, it is sufficient to solve its scenario-based robust counterpart (A2.5)-(A2.6) while selecting $\mathrm{N}_{\mathrm{s}}$ based on inequality (A2.7).

In the scenario-based robust optimization problem (5)-(10), all optimization variables belonging to $\psi$ can be linearly expressed based on $r_{h t s}^{\mathrm{DG}, \mathrm{P}}, r_{h t s}^{\mathrm{DG}, \mathrm{Q}}$ and $r_{k t s}^{\mathrm{SG}, \mathrm{Q}}$. Accordingly, for this problem, $\mathrm{N}_{\mathrm{v}}$ can be calculated as:

$\mathrm{N}_{\mathrm{v}}=2 \mathrm{~N}^{\mathrm{DG}}+\mathrm{N}^{\mathrm{SG}}$,

where $\mathrm{N}^{\mathrm{DG}}$ and $\mathrm{N}^{\mathrm{SG}}$ are the number of DDGs and SDGs installed in the ADN, respectively.

\section{REFERENCES}

[1] E. Scolari, F. Sossan, and M. Paolone, "Irradiance prediction intervals for PV stochastic generation in microgrid applications," Solar Energy, vol. 139, pp. 116-129, Dec. 2016

[2] Y. G. Rebours, D. S. Kirschen, M. Trotignon, and S. Rossignol, "A survey of frequency and voltage control ancillary services-Part I: Technical features," IEEE Trans. Power Syst., vol. 22, no. 1, pp. 350-357, Feb. 2007.

[3] "Grid integration of large capacity renewable energy sources and use of large-capacity electrical energy storage," Geneva, Switzerland, Int. Electrotech. Commission, White Paper, Oct. 2012.

[4] M. Cailliau et al, Integrating Intermittent Renewables Sources in the EU Electricity System by 2020: Challenges and Solutions, Eurelectric, Brussels, Belgium, 2010.

[5] M. Heleno, R. Soares, J. Sumaili, R. J. Bessa, L. Seca, and M. A. Matos, "Estimation of the flexibility range in the transmission-distribution boundary," in Proc. IEEE Eindhoven Power Tech, Jun./Jul. 2015 pp. 1685-1691.

[6] F. Capitanescu, "TSO-DSO interaction: Active distribution network power chart for TSO ancillary services provision," Elect. Power Syst. Res., vol. 163, pp. 226-230, Oct. 2018.

[7] J. P. Silva et al., "Estimating the active and reactive power flexibility area at the TSO-DSO interface," IEEE Trans. Power Syst., vol. 33, no. 5, pp. 4741-4750, Sep. 2018.

[8] J. Silva, J. Sumaili, R. J. Bessa, L. Seca, M. A. Matos, and V. Miranda, "The challenges of estimating the impact of distributed energy resources flexibility on the TSO/DSO boundary node operating points," Comput. Oper. Res, vol. 96, pp. 294-304, Aug. 2018.

[9] H. Gerard, E. Rivero, and D. Six, Basic Schemes for TSO-DSO Coordination and Ancillary Services Provision, Smartnet, 2016. [Online]. Available: http://smartnet-project.eu/wpcontent/uploads/2016/12/D1.3_20161202_V1.0.pdf

[10] P. Mandatova and O. Mikhailova, Flexibility and Aggregation Requirements for Their Interaction in the Market, Eurelectric, Brussels, Belgium, 2014. [Online]. Available: https://www.usef.energy

[11] A. Bernstein and E. Dall'Anese, "Linear power-flow models in multiphase distribution networks," in Proc. 7th IEEE Int. Conf. Innovat. Smart Grid Technol., Sep. 2017, pp. 1-8.

[12] A. Cabrera-Tobar, E. Bullich-Massagué, M. Aragüés-Peñalba, and O. Gomis-Bellmunt, "Capability curve analysis of photovoltaic generation systems," Solar Energy, vol. 140, pp. 255-264, Dec. 2016.

[13] P. Kundur, Power System Stability and Control. New York, NY, USA: McGraw-Hill, 1994.

[14] S. Engelhardt, I. Erlich, C. Feltes, J. Kretschmann, and F. Shewarega, "Reactive power capability of wind turbines based on doubly fed induction generators," IEEE Trans. Power Electron., vol. 26, no. 1, pp. 364-372, Mar. 2011.

[15] M. C. Campi and S. Garatti, "The exact feasibility of randomized solutions of uncertain convex programs," SIAM J. Optim., vol. 19, no. 3, pp. 1211-1230, 2008. 
[16] M. E. Baran and F. F. Wu, "Network reconfiguration in distribution systems for loss reduction and load balancing," IEEE Trans. Power Del., vol. 4, no. 2, pp. 1401-1407, Apr. 1989.

[17] Homer Energy. [Online]. Available: http://homerenergy.com/

[18] M. Bozorg, F. Sossan, J.-Y. Le Boudec, and M. Paolone, "Influencing the bulk power system reserve by dispatching power distribution networks using local energy storage," Elect. Power Syst. Res., vol. 163 pp. 270-279, Oct. 2018.

[19] M. Kalantar-Neyestanaki, M. Bozorg, F. Sossan, and R. Cherkaoui, "Allocation of frequency control reserve from aggregated resources of active distribution systems," in Proc. Power Syst. Comput. Conf., Dublin, Ireland, Jun. 2018, pp. 1-8.

[20] Gurobi Optimizer Reference Manual, Gurobi Optim., Inc., Beaverton, OR, USA. [Online]. Available: http://www.gurobi.com

[21] J. Löfberg, "YALMIP: A toolbox for modeling and optimization in MATLAB," in Proc. IEEE Int. Conf. Robot. Autom., New Orleans, LA, USA, 2004, pp. 284-289.

[22] H. R. Baghaee, M. Mirsalim, G. B. Gharehpetian, and H. A. Talebi, "Reliability/cost based multi-objective Pareto optimal design of standalone wind/PV/FC generation microgrid system," Energy, vol. 115, no. 1, pp. 1022-1041, Nov. 2016.

[23] H. R. Baghaee, M. Mirsalim, G. B. Gharehpetian, and A. K. Kaviani, "Security/cost-based optimal allocation of multi-type FACTS devices using multi-objective particle swarm optimization," Simulation, vol. 88 , no. 8, pp. 999-1010, Aug. 2012

[24] L. Bam and W. Jewell, "Review: Power system analysis software tools," in Proc. IEEE Power Eng. Soc. Gen. Meeting, San Francisco, CA, USA, 2005, pp. 139-144.

[25] S. Boyd and L. Vandenberghe, Convex Optimization. Cambridge, U.K.: Cambridge Univ. Press, 2004.

[26] G. C. Calafiore and M. C. Campi, "The scenario approach to robust control design," IEEE Trans. Autom. Control, vol. 51, no. 5, pp. 742-753, May 2006.

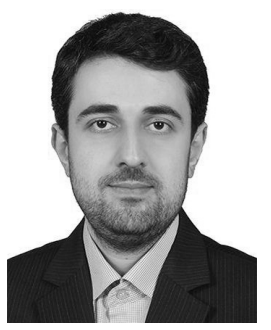

Mohsen Kalantar-Neyestanaki (S'14) received the B.Sc. degree (Hons.) in electrical engineering and physics, and the M.Sc. degree (Hons.) in electrical engineering from the Sharif University of Technology, Tehran, Iran, in 2011 and 2013, respectively. He is currently pursuing the Ph.D. degree with the Power System Group, École Polytechnique Fédérale de Lausanne, Lausanne, Switzerland. He worked as a Senior Researcher with Power Systems Research Group, Sharif University of Technology, from 2013 to 2015. From 2015 to 2016, he worked as a R\&D Engineer with the Wide Area Measurement System Control Center, Iran Grid Management Company (the TSO of Iran), Tehran. His research interests include modernizing power systems with particular reference to the integration of distributed storage and energy resources, numerical optimization and modeling techniques for power systems control, operation and planning, and wide-area monitoring, protection and control systems.

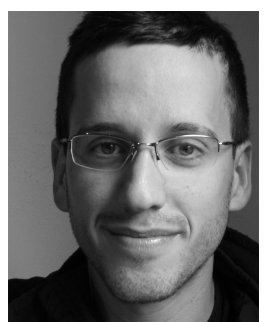

Fabrizio Sossan received the master's degree in computer engineering from the University of Genova in 2009, and the Ph.D. degree in electrical engineering from the Technical University of Denmark with a thesis on control of flexible demand to provide services to the power grid in 2014. From 2014 to 2018, he was a Post-Doctoral Fellow with the Distributed Electrical Systems Laboratory, École Polytechnique Fédérale de Lausanne, Lausanne, Switzerland, where he worked on control of battery energy storage systems and forecasting of PV generation. In 2018, he was a Guest Scientist with NREL, USA, and a Scientist with the Supply of Electricity Program, ETH, Switzerland. He is currently an Associate Professor with the Centre for Processes, Renewable Energies and Energy Systems, Mines ParisTech, France. His research interests focus on control, scheduling, and planning of distributed storage and energy resources to achieve fully sustainable power grids.

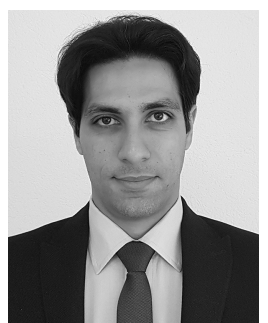

Mokhtar Bozorg received the B.Sc. and M.Sc. degrees in electrical engineering from the Sharif University of Technology, Tehran, Iran, in 2008 and 2011, respectively, and the Ph.D. degree from the École Polytechnique Fédérale de Lausanne (EPFL), Lausanne, Switzerland, in 2015, where he joined the Power System Research Group in 2011. From 2015 to 2018, he was a Post-Doctoral Fellow and the Guest Scientist with Distributed Electrical System Laboratory, EPFL. Since 2019, he has been an Associate Professor in energy and power systems with the University of Applied Sciences Western Switzerland. His research interest includes smart grids and active distribution networks, applications of mathematical modeling, optimization techniques, data analytics in power system operation and planning, and Integration of renewable energy sources and energy storage systems into power systems.

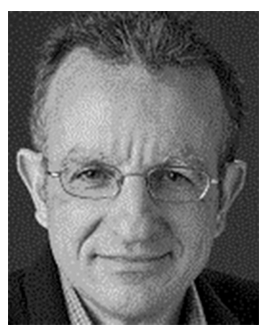

Rachid Cherkaoui (M'05-SM'07) received the M.Sc. and Ph.D. degrees in electrical engineering from the Swiss Federal Institute of Technology in Lausanne (EPFL), Switzerland, in 1983 and 1992, respectively.

$\mathrm{He}$ is currently a Senior Scientist with EPFL, Leading the Power Systems Group. He has authored and coauthored of more than 100 scientific publications. His research interests are in electricity market deregulation, distributed generation and storage, and power system vulnerability mitigation. He is a member of technical program committees of various conferences and was member of CIGRE TF's and WG's. He was an IEEE Swiss Chapter Officer from 2005 to 2011. 